\title{
Editorial
}

\section{The impact of Covid 19 on perinatal care}

Since being declared a pandemic in March 2020 by the World Health Organization (WHO), the novel coronavirus disease (COVID-19) has rapidly spread across the globe. As of November 2021, there have been nearly 248 million confirmed cases and more than5 million deaths worldwide $^{(1)}$. These staggering figures have resulted in an array of public health, social, and economic issues impacting the daily life and disrupting the social interactions and free movement of the global popullation. In Sri Lanka also the situation was no different. The number of Covid 19 cases reported in Sri Lanka up to date is 542000 and the number of deaths reported being $13760^{(2)}$. These numbers reflect the challenging nature of the Covid 19 and the enormous strain on the healthcare system in any country. The impact on the vulnerable group such as elderly, differently able people, pregnant mothers and children need more attention and planning of provision of care.

The Covid-19 pandemic impacts reproductive and perinatal health both directly through disease transmission itself but also indirectly as a consequence of changes in health care, social policy, or social and economic circumstances. The direct and indirect consequences of COVID-19 on maternal health are intertwined.

Pregnant women who have symptomatic COVID-19 may experience more severe outcomes than people who are not pregnant.In Sri Lanka although we have not experienced any deaths among pregnant women in year 2020, we have seen accumulated number of 57 deaths in this group during the last eight months.
According to $\mathrm{WHO}$, transmission in utero and through breast milk are uncommon.In infants, the risk of COVID-19 infection is usually low, and is typically mild or asymptomatic, while the consequences of not breastfeeding and separation between mother and child can be significant ${ }^{(3)}$. The guidelines for labour, delivery, and breastfeeding for COVID-19 positive patients vary, and this variability could create uncertainty and unnecessary harm. Prenatal care visits decreased, healthcare infrastructure was strained, and potentially harmful policies are implemented with little evidence in high and low/middle income countries.

The social and economic impact of COVID-19 on maternal health is marked. A high frequency of maternal mental health problems, such as clinically relevant anxiety and depression, during the epidemic are reported in many countries. This likely reflects an increase in problems, but studies demonstrating a true change are lacking. Domestic violence appeared to be increased. Women were more vulnerable to losing their income due to the pandemic than men, and working mothers struggled with increased childcare demands ${ }^{(4)}$.

More resources should be directed to epidemiological studies, health and social services for pregnant women and mothers should not be diminished, and more focus on maternal mental health during the epidemic is needed.

In Sri Lanka, the Ministry of Health took stringent actions to maintain essential maternal and child health services including services for pregnant women who are infected with COVID, suspected or quarantined amidst the pandemic situation. The country's well-structured maternal 
death surveillance response was functioning optimally even during the pandemic and all probable maternal deaths were captured, reviewed and appropriate remedial actions were taken at all levels by the Ministry of Health. Vaccination of the pregnant mothers with risk factors were initiated in June 2021. Initially Sinopharm vaccine was recommended. Now any vaccine can be given during the pregnancy and booster dose would be given in the subsequent pregnancies ${ }^{(5)}$. These measures have dramatically brought down the maternal mortality due to Covid 19 infection.

There are few infant deaths due to Covid 19 infection and those deaths have been reviewed at central level. For the first time few cases were reported having neonatalmulti system inflammatory syndrome (MIS- N). One case was reported as an immune mediated probably related to maternal vaccination.

These facts reemphasize the importance of surveillance, data collection and analysis and making decisions and development of policies based on data. All the stakeholders should collaborate and work together efficiently and effectively to achieve best results against Covid 19. The decisions taken at the board rooms should yield results at grass root level relieving the burdens on common masses.

\section{References}

1. Coronavirus disease (COVID-19) Weekly Epidemiological Update and Weekly Operational Update, WHO. https://www.who.int/emergencies/dise ases/novel-coronavirus-2019/situationreports

2. COVID-19: Live Situational Analysis Dashboard of Sri Lanka-Health Promotion Bureau.

https://hpb.health.gov.lk/covid19dashboard/

3. Breast feeding and Covid 19, Scientific Brief, WHO. June 2020. https://www.who.int/newsroom/commentaries/detail/breastfeedin g-and-covid-19

4. Kotlar B, Gerson, E, Petrillo, S. et al. The impact of the COVID-19 pandemic on maternal and perinatal health: a scoping review. Reprod. Health18, 10 (2021). https://doi.org/10.1186/s12978-02101070-6

5. Covid 19 Guidelines -Family Health Bureau. https://fhb.health.gov.lk/index.php/en/ covid-19

\section{SuranthaPerera Managing Editor}

